\title{
ANÁLISE CRÍTICA E COMPARATIVA ENTRE LEGISLAÇÃO E OS CUIDADOS DOS IDOSOS RESIDENTES EM INSTITUIÇÕES DE LONGA PERMANÊNCIA PARA IDOSOS
}

\author{
Gerídice Lorna Andrade de Moraes' \\ ORCID: 0000-0002-7097-0841 \\ Maria Célia de Freitas" \\ ORCID: 0000-0003-4487-1193
}

Maria Josefina da Silva'"I

ORCID 0000-0003-4866-9224

Rachel Gabriel Bastos Barbosa"I

ORCID: 0000-0003-4205-6910

Janaina Fonseca Victor Coutinho"'I

ORCID: 0000-0001-7451-0132

Marília Marques Braga"'

ORCID: 0000-0002-7483-1435

'Prefeitura Municipal de Fortaleza, Secretaria Municipal de Saúde. Fortaleza, Ceará, Brasil.

"Universidade Estadual do Ceará. Fortaleza, Ceará, Brasil.

"'Universidade Federal do Ceará. Fortaleza, Ceará, Brasil.

Autor Correspondente: Gerídice Lorna Andrade de Moraes E-mail: geridice@hotmail.com

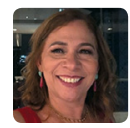

Como citar:

Moraes GLA, Freitas MC, Silva MJ, Barbosa RGB, Coutinho JFC, Braga MM. Análise Crítica e

Comparativa entre Legislação e os Cuidados dos Idosos Residentes em Instituições de Longa Permanência para Idosos. In: Santana RF (Org.). Enfermagem gerontológica no cuidado do idoso em tempos da COVID 19. Brasilia, DF: Editora ABen;

2021. 171 p. (Serie Enfermagem e Pandemias, 5). https://doi.org/10.51234/aben.21.e05.c03

\section{INTRODUÇÃO}

As mudanças demográficas e epidemiológicas a partir da segunda metade do século passado, decorrentes dos avanços dos conhecimentos no campo das ciências da saúde, impõem intervenções do Estado, da sociedade como um todo e de organizações civis no sentido de prover condições de suporte a este novo contingente populacional, o idoso.

O século $X X$ trouxe mudanças em todos os aspectos da vida humana com uma rapidez e profundidade nunca vivida. Até a geração anterior a dos nossos avós cada família era responsável por seus idosos, que não exigiam muitos cuidados uma vez que a expectativa de vida era curta. $\mathrm{O}$ tempo de vida se prolongou, a pirâmide populacional sofreu alterações em seus contornos, as famílias reduziram em número de membros e nas relações parentais, as pessoas sofreram mobilidade por motivos de trabalho, casamento ou a busca de uma vida melhor; o cotidiano se transformou em um turbilhão de tarefas para a satisfação das necessidades cada vez maiores.

Dessa forma, os espaços institucionais que proporcionavam abrigos em ambientes extra familiar existem ao longo da história, mas somente em tempos recentes foram criadas instituições para abrigar especificamente idosos, considerando estes aspectos relacionados e outros, como a incapacidade da família em cuidar do idoso dependente.

Data em torno de 130 anos o surgimento da primeira iniciativa de abrigar idosos, o Asilo São Luiz para a Velhice Desamparada, criado em 1890. Ao longo desse tempo muitas foram as transformações nesse aspecto, especialmente pela pressão populacional e de direitos estabelecidos para esta faixa etária. A nova denominação dada aos asilos, Instituições de longa Permanência para Idosos - ILPI é normatizada pela Resolução 283, de 23 de setembro de 2005 pela Anvisa - Agencia Nacional de Vigilância Sanitária, com caráter residencial, destinada a 
domicílio coletivo de pessoas com idade igual ou superior a 60 anos que, segundo a Política Nacional do Idoso, é considerado idoso(1).

Destaque-se que a regulamentação situa a ILPI como tendo caráter residencial, sem a inclusão de profissionais de saúde em seus quadros, apenas cuidadores treinados para cuidados simplificados de ajuda aos idosos residentes. Embora na descrição sobre o item saúde, a Resolução considere a inclusão de residentes idosos em condições de adoecimento, incidentes e prevalentes e ações de promoção, proteção e prevenção de saúde.

Com o aumento exponencial da longevidade, inclusive para os idosos que residem nessas instituições surge a busca à estas instituições, cada vez maior como apoio para idosos dependentes, em especial, graus II e IIII. Esses exigem cuidados complexos que a família não tem suporte para efetuá-los. Nestes casos demanda a necessidade de profissionais qualificados para realizar o cuidado, especialmente a equipe de enfermagem, consoante a legislação que orienta este grupo profissional(2).

Neste sentido, o Conselho Federal de Enfermagem (COFEN) atento a este novo espaço de prática profissional aprova a Resolução 620/2019 normatizando as atribuições próprias da equipe de enfermagem que exercem atividades no contexto das ILPs. Todavia, a Resolução da Agência Nacional de Vigilância Sanitária (Anvisa) não contempla estes aspectos operacionais do cuidado, deixando uma lacuna importante para a elaboração de regulamentação da prática de Enfermagem nestas instituições pelos órgãos responsáveis por esta normatização ${ }^{(3)}$.

Assim, faz-se necessário a análise comparativa do que estabelece a legislação geral de saúde para as ILPs e as demandas de cuidados de enfermagem e saúde, considerando a situação de promoção de saúde e prevenção da infecção por coronavirus (SARS-COV2) e, ainda, a complexidade dos cuidados no manejo aos idosos residentes nas instituição de longa permanência.

Espera-se com isto identificar pontos relevantes que possam colaborar na formulação de políticas e normas operacionais voltadas ao bem-estar do idoso residente em ILPIs de modo a garantir o que está posto no Estatuto do Idoso, Art. 9. “É obrigação do Estado, garantir à pessoa idosa a proteção à vida e à saúde, mediante efetivação de políticas sociais públicas que permitam um envelhecimento saudável e em condições de dignidade"(4).

\section{OBJETIVO}

Realizar uma análise crítica e comparativa do que estabelece a legislação geral de saúde para as Instituição de Longa Permanência para Idosos e a equipe de enfermagem frente as transformações relativas aos cuidados dos residentes nestas instituições.

Com base nas evidências existentes, o vírus COVID-19 é transmitido entre pessoas por meio do contato próximo e de gotículas, e não é transmitido pelo ar. As pessoas com maior risco de desenvolver a infecção são os idosos, pois, além de fatores como comorbidades e resposta imunológica mais demorada, entram em contato próximo com profissionais de saúde, cuidadores, familiares e os demais residentes ${ }^{(5)}$.

Neste sentido, políticas a serem estabelecidas para as ILPI devem contar com a participação dos enfermeiros e seus órgãos de classe considerando a legislação profissional e a necessidade permanente de cuidados de média e alta complexidade hoje presentes nas ILPIs. Esta não é a realidade expressa na legislação, corroborando a necessidade do presente estudo.

\section{MÉTODO}

Estudo comparativo dos documentos que determinam a legislação geral da saúde para as Instituições de Longa Permanência, a saber: Resolução 283/2005 da ANVISA, Nota Técnica GVIMS/GGTES/ANVISA No 05/2020 e Resolução 620/2019 do Conselho Federal de Enfermagem que normatiza as ações para equipe de Enfermagem nas referidas instituições ${ }^{(3,6)}$.

Além dos documentos citados, utilizou-se artigos para fundamentar a reflexão e análise crítica dos aspectos relevantes ao estudo. 


\section{RESULTADOS}

Determinações do dimensionamento de Enfermagem as Instituições de Longa Permanência para Pessoas Idosas- ILPI nos documentos oficiais na legislação em tempos de COVID-19.

RESOLUÇÃO - RDC No 283, DE 26 DE SETEMBRO DE 2005

Estabelece o padrão mínimo de funcionamento das Instituições de Longa Permanência para Idosos.

A Instituição de Longa Permanência para Idosos deve possuir um Responsável Técnico - RT pelo serviço, que responderá pela instituição junto à autoridade sanitária local.

O Responsável Técnico deve possuir formação de nível superior.

\section{Recursos Humanos}

A Instituição de Longa Permanência para Idosos deve apresentar recursos humanos, com vínculo formal de trabalho, que garantam a realização das seguintes atividades:

Para a coordenação técnica: Responsável Técnico com carga horária mínima de 20 horas por semana.

Para os cuidados aos residentes:

a) Grau de Dependência I: um cuidador para cada 20 idosos, ou fração, com carga horária de 8 horas/dia;

b) Grau de Dependência Il: um cuidador para cada 10 idosos, ou fração, por turno; c) Grau de Dependência IIl: um cuidador para cada 6 idosos, ou fração, por turno.

Para as atividades de lazer: um profissional com formação de nível superior para cada 40 idosos, com carga horária de 12 horas por semana.

Para serviços de limpeza: um profissional para cada $100 \mathrm{~m} 2$ de área interna ou fração por turno diariamente.

Para o serviço de alimentação: um profissional para cada 20 idosos, garantindo a cobertura de dois turnos de 8 horas.

Para o serviço de lavanderia: um profissional para cada 30 idosos, ou fração, diariamente.

A instituição que possuir profissional de saúde vinculado à sua equipe de trabalho, deve exigir registro desse profissional no seu respectivo Conselho de Classe.

A Instituição deve realizar atividades de educação permanente na área de gerontologia, com objetivo de aprimorar tecnicamente os recursos humanos envolvidos na prestação de serviços aos idosos.

\section{RESOLUÇÃO COFEN № $620 / 2019$}

NOTA TÉCNICA GVIMS/

GGTES/ANVISA No 05/2020

Normatiza as atribuições dos Profissionais de Enfermagem nas Instituições de Longa Permanência para Idosos - ILPI.

Enfermeiros:

São atribuições do Enfermeiro na Instituições de Longa Permanência para Idosos (ILPI):

Exercer a função assistencial com atenção integral voltada para a promoção, proteção e recuperação da saúde do idoso;

Coordenar e supervisionar as ações desenvolvidas pelos técnicos e auxiliares de enfermagem;

Realizar o acolhimento do idoso e de sua família, incentivando a participação da família no cuidado;

Desenvolver ações para a manutenção e fortalecimento do vínculo familiar e/ou representante legal dos idosos institucionalizados;

Implementar e realizar a consulta e prescrição de enfermagem através do processo de enfermagem, ao idoso na ILPI, utilizando o olhar da Avaliação Geriátrica Ampla (AGA);

Determinar ações que possam atender integralmente às necessidades biopsicossociais e espirituais dos idosos residentes;

Avaliar o idoso por meio de escalas específicas contemplando, sobretudo, a funcionalidade global (cognição, humor, aspectos físicos, psicológicos, mobilidade e comunicação) e as atividades de vida diária, classificando os riscos à saúde do idoso;

Desenvolver plano de cuidados personalizado, mantendo e estimulando a autonomia e a independência funcional dos idosos residentes;

Respeitar o direito da pessoa idosa quanto ao exercício da sua sexualidade;

Promover a saúde dos residentes por meio de ações, tais como a imunização e a implantação de rotinas de prevenção de agravos;

Ofertar cuidados paliativos precocemente, nas situações em que forem pertinentes;

Trabalhar em uma abordagem multiprofissional e interdisciplinar;

Contribuir na avaliação anual do plano de atenção integral à saúde;

Participar da elaboração dos Protocolos Operacionais Padrão (POPs);

Registrar no prontuário do paciente e em outros documentos padronizados as informações inerentes e indispensáveis ao processo de cuidar de forma clara, objetiva, cronológica, legível, completa e sem rasuras.
Orientação para a prevenção e o controle de infecções pelo novo coronavírus (sars-cov-2) em Instituições de Longa permanência para idosos (ILPI).

\section{Profissionais/Cuidadores}

As ILPIs devem implementar políticas de licença médica, que não sejam punitivas, para permitir que profissionais e cuidadores que apresentem sintomas de infecção respiratória, fiquem em casa.

Profissionais e cuidadores que tenham contato com pessoas com sintomas de infecções respiratórias ou contato com pessoas sabidamente com COVID-19, fora da instituição, também não devem ter contato com os residentes ou circular nas mesmas áreas que estes.

Orientar os funcionários para a realização correta e frequente da higiene das mãos com água e sabonete líquido OU álcool gel a $70 \%$, de acordo com as recomendações da Anvisa, incluindo antes e depois do contato com os residentes e com seus dormitórios, após contato com superfícies ou equipamentos potencialmente contaminados e após remover os equipamentos de proteção individual (EPI).

Fornecer orientações atualizadas sobre a COVID-19 para profissionais/cuidadores e familiares sobre a COVID-19, reforçando a necessidade da adoção de medidas de prevenção e controle dessas infecções.

Restringir a visita de profissionais que prestam serviços periódicos e voluntários, como, por exemplo, cabeleireiros, podologistas, grupos religiosos, etc. Caso seja estritamente necessário, a ILPI deve certificar-se que nenhuma dessas pessoas apresente sintomas de infecção respiratória, antes de mesmo de que essas pessoas entrem em contato com os idosos. 


\section{ANÁLISE CRITICA}

As políticas que orientam o funcionamento das ILPI's foram orientadas no cenário de um país ainda despreparado para as mudanças epidemiológicas, demográficas e sociais com relação ao idoso e para a população que recorre às ILPIs como recurso para ser cuidado. Assim, há uma grande necessidade de se repensar as Políticas públicas com foco nas Instituições de longa permanência para idosos.

Como parte integrante dos recursos institucionais para o atendimento do idoso, segundo o que determina o Estatuto do Idoso, as ILPIs ainda carecem de atender as necessidades dos idosos na sua integralidade. Segundo o Estatuto do Idoso faz-se necessário a participação de equipe multidisciplinar para atender as demandas do idoso e, no cenário das ILPIs a enfermagem Gerontológica tem um papel essencial no cuidado integral ao idoso institucionalizado. O desafio de implantar e implementar esta integralidade do cuidado à pessoa idosa institucionalizados é complexo pois os documentos existentes não refletem o avanço neste campo, quanto ao perfil do idoso institucionalizado e a complexidade do cuidado no contexto da institucionalização nos dias atuais.

Embora o documento da Agência de Vigilância Sanitária (ANVISA) - Resolução n²83 de 26 de setembro de 2005, que disciplina o Regimento Interno para funcionamento estabelecendo um padrão mínimo de funcionamento e avaliação de Instituições de Longa Permanência para idosos necessite ser analítico, apenas aborda os processos de forma geral: resumidamente fala em um responsável técnico com requisito de ser graduado; fala de recursos humanas com vínculos formais para o cuidado aos idosos nos três gradientes de dependência (I,II e III), estabelece a presença de um profissional de saúde com vínculo, sem detalhar a forma desta vinculação (se formal, como funcionário ou prestador de serviços eventuais) e destaca a necessidade de educação permanente em gerontologia, sem ,contudo, evidenciar aspectos relevantes desta abordagem. Inicia definindo o cuidador como uma pessoa capacitada para auxiliar o idoso que apresenta limitações nas atividades de vida diáriaa ${ }^{(6)}$.

Considerando que as ILPIs abrigam idosos com todos os níveis de dependência, qual seria o perfil do profissional de saúde mais adequado para o cuidado em cada um destes níveis bem como para a capacitação desses cuidadores? Na seção de recursos humanos, o documento descreve a necessidade de responsável técnico, determinando nível superior, mas não define as competências nem a necessidade de qualificação em saúde do idoso. Define ainda a quantidade de cuidadores necessários de acordo com o grau de dependência do idoso institucionalizado. Aqui surge outro ponto importante: qual o profissional com as competências e habilidades necessárias para fazer esta classificação de acordo com o grau de dependência e avaliação de sua funcionalidade no seu processo de ingresso na instituição?

Vale ressaltar que no contexto das ILPI's outros serviços também precisam ser oferecidos, como por exemplo, serviços de limpeza, de orientação e acompanhamento nutricional, lavanderia, hotelaria, fisioterapia, terapia ocupacional, assim como atividades de lazer. A atribuição de tais atividades certamente não é papel de apenas um profissional, mas sim de uma equipe multidisciplinar especializada envolvida no cuidado.

As instituições de longa permanência (ILPs), como casas de repouso e centros de reabilitação, são organizações que cuidam de pessoas que sofrem de incapacidade física ou mental, algumas das quais em idade avançada. Pessoas que vivem em ILPs são populações vulneráveis, que têm um risco maior de infecção e desfechos adversos por viverem muito próximos de outras pessoas. Sendo assim, as ILPs devem tomar precauções especiais para proteger seus residentes, funcionários e visitantes. Observe que as atividades de prevenção e controle de infecção $(\mathrm{PCl})$ podem afetar a saúde mental e o bem-estar dos residentes e funcionários, especialmente o uso de EPIs e a restrição de visitantes e atividades em grupo.

A Resolução 283/ANVISA não inclui a presença em seu quadro permanente de tais profissionais no cuidado ao idoso institucionalizado, como por exemplo a presença do enfermeiro, prestadora de cuidados contínuos necessitando, portanto, de período integral nas instituições. 
Na Nota Técnica, № 05/2020, atualizada em 06 de 2020, as orientações para prevenção e o controle de infecção pelo novo coronavirus (SARS-Cov-2) em ILPI, estabelece orientações mínimas para as ILPIs quanto às medidas de prevenção e controle que devem ser adotadas durante a assistência aos residentes, principalmente com relação aos casos suspeitos ou confirmados de COVID- 19. As orientações são fundamentadas nas orientações divulgadas pela Organização Mundial da Saúde (OMS) e pelos Centers for Disease Control and Prevention (CDC/EUA). Em todas as diretrizes não define qual o profissional responsável para a implementação das referidas medidas no cotidiano das ILPs ${ }^{(7)}$.

No entanto, é o enfermeiro que organiza as ações cuidativas de modo a promoção atividades educativas para esclarecimento dos residentes e profissionais sobre medidas protetivas, bem como as ações necessárias a condução com os idosos do local.

As Instituições de Longa Permanência para Idosos (ILPIs) possuem uma população de residentes que, em geral, são mais vulneráveis, com níveis variados de dependência e com necessidades complexas. Sendo assim, as ILPIs devem implementar medidas de prevenção e controle de infecção para evitar ou reduzir ao máximo que os residentes, seus cuidadores e demais profissionais que atuam nesses estabelecimentos sejam infectados pelo novo coronavírus (SARS-CoV-2) e, mais significativamente, para reduzir a morbimortalidade entre os idosos dessas instituições ${ }^{(7)}$.

As medidas de prevenção que devem ser aplicadas são similares às medidas para detectar e impedir a propagação de outros vírus respiratórios, como, por exemplo, o vírus da influenza. Destaque-se que a Resolução 283/2005, que orienta o funcionamento das ILPIs não determina o dimensionamento de pessoal de enfermagem nas ILPI's.

É fato que diante da pandemia por COVID-19 são necessárias intervenções efetivas para o controle da doença com implementação de ações de prevenção como: estratégias de isolamento social e suspensão de visitas, orientação sobre a doença e ações de prevenção como lavagem das mãos e uso de álcool 70\% para profissionais envolvidos no cuidado, detecção precoce e isolamento de idosos com suspeita COVID -19, além de construção de fluxograma interno durante a pandemia. O corpo de funcionários de enfermagem nestas instituições pode colaborar neste mister. Quando se aborda o problema dos diferentes graus de complexidade de dependência dos residentes fica evidente a necessidade da equipe de enfermagem para a realização dos cuidados, supervisão dos mesmos e o planejamento do cuidado, competência exclusiva do enfermeiro ${ }^{(6)}$

As ILPIs, como cenário de prática acadêmica do futuro enfermeiro, também precisam ter, pela legislação que regulamenta a formação profissional, a presença do enfermeiro da instituição no acompanhamento dos discentes, mesmo que junto deles esteja o docente da instituição de ensino. Por fim, ações de educação permanente nas ILPIs vai requerer um profissional capacitado em técnicas específicas de educação em saúde e em gerontologia, inclusive no que respeita a cuidados ao idoso e o enfermeiro tem formação acadêmica para esta atribuição.

Frente a esta realidade o Conselho Federal de Enfermagem se defronta com a necessidade de regulamentar o papel do enfermeiro e a equipe de enfermagem e estabelece a Resolução no620/2019 que normatiza as atribuições dos Profissionais de Enfermagem nas Instituições de Longa Permanência para idosos. Nele considera a inserção dos cuidados de enfermagem nas ILPI's trazendo várias outras resoluções fundamentais para a melhoria da qualidade do cuidado de enfermagem nessas instituições, como o Código de Ética de Profissionais de Enfermagem, de registros das ações profissionais eletrônico ou tradicionais nas ILPI's, a implantação da Sistematização de Enfermagem, a necessidade de qualificação profissional nas instituições de forma integral numa perspectiva multidimensional e ações de segurança para o paciente, profissional e instituições de saúde ${ }^{(3)}$.

A Resolução descreve as atribuições da equipe de enfermagem necessárias nas ILPI's e o papel do enfermeiro tanto como responsável técnico como exercendo função assistencial à pessoa idosa institucionalizada ${ }^{(7)}$.

Logo, a premissa do cuidado multidimensional ao idoso, com diferentes escalas para avaliação integral do idoso, para atender as sus especificidades, necessário de faz um profissional com competência clínica e conhecimento do significado de cada instrumento de avaliação do idoso para seu uso adequado e o 
planejamento do cuidado personalizado. O Enfermeiro domina estas técnicas pois em sua formação acadêmica são estudadas e aplicadas nos cenários de prática.

Nessa perspectiva, o profissional de enfermagem deve refletir sobre o seu papel no funcionamento e organização da ILPI's numa perspectiva integral com foco na promoção, proteção e recuperação da saúde do idoso. Observa-se que as orientação contidas na Nota Técnica aqui referida são dirigidas para gestores de ILPs, objetivando oferecer orientações sobre prevenção da doença nas ILPS, dentro do contexto da COVID-19 para: 1) evitar que o vírus entre na instituição, 2) evitar que o vírus se espalhe na instituição, e 3) evitar que o vírus se dissemine para fora da instituição( ${ }^{(5)}$.

Os documentos que regulamentam as ILPI's não acompanham a complexidade do cuidado ao idoso que só se amplia e que é refletida pelo aumento da demanda e procura de instituições de longa permanência por familiares de idosos com doenças crônicas-degenerativas e incapacitantes e que buscam cuidado mais especializado. A carga horária dos enfermeiros deve ser considerada conforme o grau de dependência e comorbidades dos idosos e, isso pode determinar a qualidade da assistência prestada na ILPI.

A Resolução do COFEN n620/2019 traz as diversas atribuições dos enfermeiros desde ações voltadas às questões biopsicossociais e de saúde até espirituais, enfatizando a importância da avaliação global da pessoa idosa e de cuidados individualizados para cada residente. Fato é que Instituições de Longa Permanência para Idosos, públicas e privadas, muitas vezes são considerados celeiros de idosos abandonados pela família sendo cuidados pela equipe de enfermagem e cuidadores ${ }^{(3)}$.

O Estatuto do Idoso (2003) tem como princípio fundamental que " a família, a sociedade e o estado têm o dever de assegurar ao idosos todos os direitos de cidadania, garantindo sua participação na comunidade, defendendo sua dignidade, bem-estar e direito à vida" e um capítulo específico à habitação que conta com dois artigos: o primeiro dispõe que "o idoso tem direito à moradia digna, no seio da família natural ou substituta, ou desacompanhado de seus familiares, quando assim o desejar, ou ainda, em instituição pública ou privada"; o segundo artigo estabelece que "A assistência integral na modalidade de entidade de longa permanência será prestada quando verificada inexistência de grupo familiar, casa-lar, abandono ou carência de recursos financeiros próprios ou da família"(4).

Portanto, impera a necessidade do enfermeiro no contexto da instituição de longa permanência de forma efetiva, desde a avaliação da necessidade de institucionalização juntamente com a assistente social, no acolhimento a esse idoso, no acompanhamento longitudinal e na reabilitação das atividades básicas e instrumentais de vida diária da pessoa idosa institucionalizada. Entretanto, a RDC de n 283 de 2005 não cita as atribuições do enfermeiro e, portanto, dado o desconhecimento das nuances deste ser idoso, os gestores não sabem dimensionar com clareza o trabalho do enfermeiro como prioritário para a assistência a pessoa idosa ${ }^{(6,8)}$.

O desafio presente é a reformulação das políticas públicas de proteção ao idoso institucionalizado, frente à vertiginosa mudança no perfil do idoso institucionalizado, seja no campo social, de saúde e de demandas e anseios pessoais. Destaca-se nesses dois documentos governamentais a fragilidade dos aspectos relativos aos cuidados de saúde complexos e contínuos, competência da equipe de enfermagem e dos enfermeiros como responsável pela prestação deste cuidado pela equipe, sendo o profissional de saúde mais efetivo nas ILPI's. O COREN, por meio da Resolução 620/2019 busca estabelecer este papel. Cabe ao órgão de classe, portanto, a fiscalização do seu cumprimento, aos profissionais de enfermagem que atuam nestas instituições demonstrar esta necessidade por meio de uma prática pautada no rigor científico e nos instrumentos da enfermagem disponíveis e, aos gestores, aos gestores a sensibilidade de fazer cumprir os preceitos constitucionais de garantias dos direitos e segurança de idosos cidadãos que estão aos seus cuidados institucionais ${ }^{(3,9)}$.

\section{Limitações do estudo}

Depara-se com uma legislação nos diferentes níveis competência em sua formulação, seja municipal, estadual e federal, que se orientam pela Legislação federal que, por sua vez, não compreende, numa perspectiva 
de cuidado de saúde e de bem-estar, o papel dos cuidados de enfermagem nas ILPI, as competência de cada membro da equipe de enfermagem, suas habilidades necessárias para o alcance das finalidades destas instituições que é garantir uma vida digna, assistida e com qualidade aos idosos que necessitam destas instituições. Assim, pouco se tem em termos de amparo na literatura de experiencias exitosas de uma prática de enfermagem planejada, com terminalidade de autônoma nas ILPs eu pudesse servir de norte para sugestões aos formuladores de políticas.

\section{Contribuições para Enfermagem Gerontológica}

Frente a realidade das ILPIs no que diz respeito a assistência à saúde dos idosos residentes, observa-se a necessidade de reformulação da legislação específica, tendo como parâmetro o que determina o COFEN para o pessoal de Enfermagem, bem como desenvolver protocolos, modelos de assistência e parâmetros para a prática de Enfermagem. Essa é uma tarefa dos que militam diretamente nestas instituições, dos órgãos de classe e, especificamente da Associação Brasileira de Enfermagem por meio do seu Departamento de Gerontologia liderar esta jornada.

\section{CONSIDERAÇÕES FINAIS}

No espaço destinado a uma reflexão não cabe uma proposta política de enfermagem para as IPLIs. Se espera que os órgãos formuladores de políticas e normas em Enfermagem sejam capazes de levar esta realidade aos formuladores de políticas públicas e uma mobilização para demonstrar empiricamente, na realidade cotidiana, a necessidade deste profissional, segundo os parâmetros de dimensionamento de pessoal e especialidade técnica. Lembrar que o idoso necessita de cuidado muito mais que terapêutica; de uma abordagem ampla, que considere seu ser como um todo que medicamentos supressores de achaques comuns ao processo de envelhecimento; de uma visão antropocêntrica que biomédica, e o enfermeiro tem desenvolvido estas habilidades ao longo de sua formação, seja na graduação, na pós-graduação strito sensu e latu sensu.

\section{REFERÊNCIAS}

1. Presidência da República (BR). Politica Nacional do Idoso [Internet]. Lei no 8.842 de 04 de janeiro de 1994 [cited 2020 Jun 28]. Available from: http://www.planalto.gov.br/ccivil_03/leis/l8842.htm

2. Agência Nacional de Vigilância Sanitária (Anvisa). Regulamento técnico para o funcionamento das instituições de longa permanência para idosos: resolução de diretoria colegiada - RDC n 283 [Internet]. 26 de setembro de 2005[cited 2020 Jun 28]. Available from: http://bvsms.saude.gov.br/bvs/saudelegis/anvisa/2005/res0283_26_09_2005.html

3. Conselho Federal de Enfermagem (COFEN). Normatiza as atribuições dos Profissionais de Enfermagem nas instituições de Longa Permanência para Idosos - ILPI [Internet]. Resolução n. 620, de 4 de novembro de 2019. Brasília, DF. DOU no 215, 06.11.2019[cited 2020 Jun 28], Seção 1, p.212. Available from: https://abmes.org.br/legislacoes/detalhe/2940/ resolucao-cofen-n-620

4. Ministério da Saúde (BR). Estatuto do Idoso. Lei no 10.741 [Internet]. 2aed. MS: Brasilia; 2007[cited 2020 Jun 28]. Available from: http://www.planalto.gov.br/ccivil_03/leis/2003/l10.741.htm

5. Organização Pan-Americana de Saúde (OPAS). Orientações sobre prevenção e controle de infecção para instituições de longa permanência no contexto da COVID-19 [Internet]. 2020[cited 2020 Jun 28]. Available from: https://iris.paho.org/ handle/10665.2/51982

6. Agência Nacional de Vigilância Sanitária (Anvisa). Nota técnica nº 05/2020- SARS-CoV-2/ANVISA - Orientações para a Prevenção e o Controle de Infecções pelo Novo Coronavírus em instituições de longa permanência para idosos (Ilpi) [Internet]. 2020[cited 2020 Jun 28]. Available from: https://www.gov.br/anvisa/pt-br/centraisdeconteudo/publicacoes/ servicosdesaude/notas-tecnicas/nota-tecnica-no-05-2020-gvims-ggtes-anvisa-orientacoes-para-a-prevencao-e-ocontrole-de-infeccoes-pelo-novo-coronavirus-em-instituicoes-de-longa-permanencia-para-idosos-ilpi.pdf/view 
7. Paula RCC, Rodrigues MA, Santana RF. Dimensionamento de pessoal de enfermagem nas instituições de longa permanência para idosos. Enferm Foco. 2018;9(1). https://doi.org/10.21675/2357-707X.2018.v9.n1.1813

8. McMichael TM, Currie DW, Clark S, Pogosjans S, Kay M, Schwartz NG. Epidemiology of Covid-19 in a Long-Term Care Facility in King County, Washington. N England J Medicine. 2020;382:2005-11. https://doi.org/10.1056/NEJMoa2005412 